\title{
MODULATION SPECTROSCOPY OF REDUCED DIMENSIONAL SEMICONDUCTOR SYSTEMS*
}

\author{
F.H. PoLLAK \\ Physics Department and New York State Center for Advanced Technology \\ in Ultrafast Photonic Materials and Application \\ Brooklyn College of the City University of New York, Brooklyn, NY 11210, USA
}

This paper reviews the background of modulation spectroscopy, particularly electromodulation, presents some recent room temperature results having both fundamental and techrological significance, including two-dimensional electron gas effects in modulation-doped, pseudomorphic $\mathrm{GaAlAs/InGaAs/GaAs} \mathrm{single} \mathrm{quantum} \mathrm{wells} \mathrm{(HEMT} \mathrm{structures),} \mathrm{quantum}$ well laser structures and process-induced damage in quantum dot arrays fabricated by reactive ion etching.

PACS numbers: 78.66.-w, 78.66.Fd, 78.40.Fy

\section{Introduction}

Modulation spectroscopy is a powerful experimental technique for studying and characterizing the properties of bulk semiconductors [1-3], reduced dimensional systems (quantum wells, superlattices, heterostructures, quantum dots, etc.) [1-3], surfaces/interfaces [1-3], growth/processing [1-3] as well as actual device structures [1, 3, 4]. Modulation spectroscopy is an analog method for taking the derivative of the optical spectrum of a material by the periodic modification of the mcasurement conditions. This procedure gives rise to sharp, differential-like spectra in the region of interband (intersubband) transitions, even at room temperature (or above). One of the most important advantages of modulation spectroscopy is the ability to perform detailed lineshape fits to extract important parameters such as interband (intersubband) energies, linewidths, Fermi energies, etc. For example, cven at $300 \mathrm{~K}$ it is possible to evaluate the energies of interband (intersubband) transitions to within a few meV. Furthermore, the effects of static external perturbations such as electric and magnetic fields, temperature, hydrostatic pressure, uniaxial stress, composition, elc. can be conveniently studied.

A particularly useful form of modulation spectroscopy is electromodulation (EM) since it is sensitive to surface/interface electric fields and can be performed

${ }^{*}$ This work was supported by the NSF, ARO and NY State Science and Technology Foundation. 
in contactless modes that require no special mounting of the sample. These techniques can be employed on wafer-sized material without altering the sample. The apparatus is simple, compact, easy to use and relatively inexpensive.

\section{Instrumentation}

Shown in Fig. 1 is a schematic drawing of the contactless EM method of photoreflectance (PR) developed at Brooklyn College [1-3]. In PR modulation of the built-in electric field of the sample is caused by photo-excited electron-hole pairs created by the pump source which is chopped/modulated at frequency $\Omega_{m}$, typically $\approx 100-200 \mathrm{~Hz}$, utilizing a mechanical chopper with maximum $\Omega_{m} \approx$ $5 \mathrm{kHz}$. Higher modulation frequencies up to about $1 \mathrm{MHz}$ can be achieved by (a) replacing the mechanical chopper by an acousto-optic modulator or (b) using an internally modulated laser diode (no chopper required).

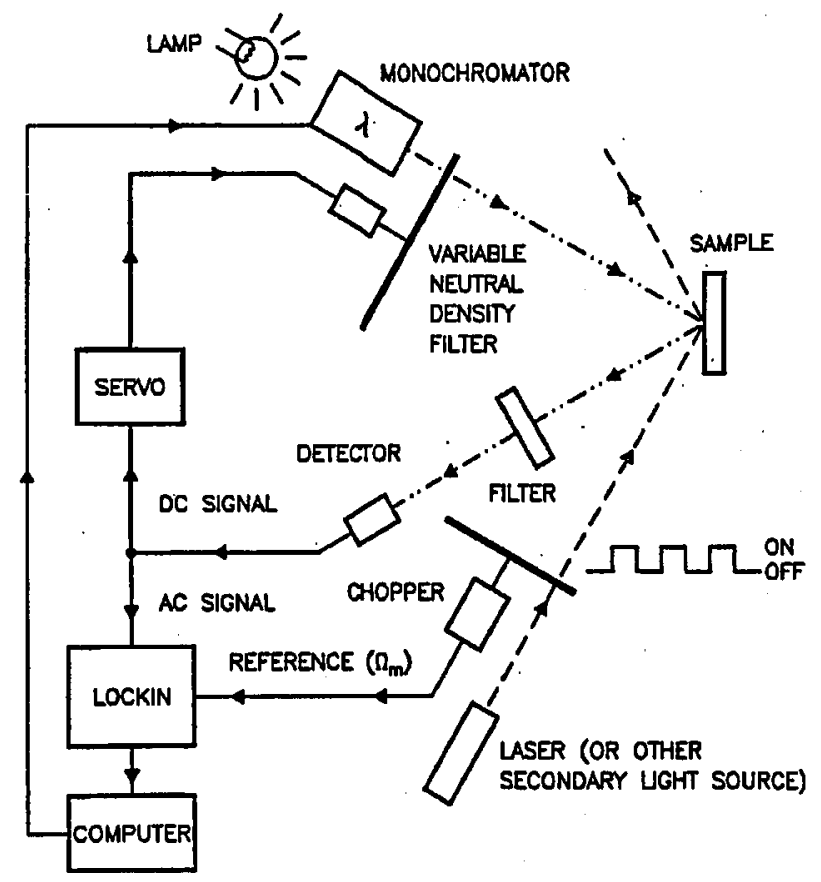

Fig. 1. Schematic representation of the Brooklyn College photoreflectance apparatus.

The light striking the detector contains two signals: the dc (or average value) is given by $I_{0}(\lambda) R(\lambda)$, where $R(\lambda)$ is the dc reflectance of the material while the modulated value (at frequency $\Omega_{m}$ ) is $I_{0}(\lambda) \Delta R(\lambda)$, where $\Delta R(\lambda)$ is the change in reflectance produced by the modulation source. The ac signal from the detector, proportional to $I_{0} \Delta R$, is measured by a lock-in amplifier (or other signal averaging procedure). Typically $I_{0} \Delta R$ is $10^{-4}-10^{-6}$ of $I_{0} R$.

In order to evaluate the quantity of interest, i.e. the relative change in reflectance $\Delta R / R$, a normalization procedure must be used to eliminate the uninteresting common feature $I_{0}(\lambda)$. Normalization is performed by a variable neutral 
density filter (VNDF) connected to a servo mechanism. The dc signal from the detector, which is proportional to $I_{0}(\lambda) R(\lambda)$, is introduced into the servo which moves the VNDF in such a manner as to keep $I_{0}(\lambda) R(\lambda)$ as a constant, i.e., $I_{0}(\lambda) R(\lambda)=C$. Under these conditions the ac signal $I_{0}(\lambda) \Delta R(\lambda)=C \Delta R(\lambda) / R(\lambda)$. Thus, the signal to the lock-in-amplifier is proportional to the quantity of interest, i.e., $\Delta R(\lambda) / R(\lambda)$.

Another contactless method of EM is contactless electroreflectance (CER) $[1,3]$. The CER method utilizes a condenser-like system consisting of a thin, transparent, conductive coating [indium-tin-oxide or 50-60 $\AA$ of a metal such as $\mathrm{Au}$ or $\mathrm{Ni}$ on a transparent substrate (glass, quartz, etc.)] which serves as one electrode. A second electrode consisting of a metal strip is separated from the first electrode by insulating spacers. The sample $(\approx 0.5 \mathrm{~mm}$ thick $)$ is placed between these two capacitor plates. Thus there is no pump beam required in CER.

An example of a contact mode of EM would be the metal-insulator(oxide)-semiconductor configuration which consists of the semiconductor, about $200 \AA$ of an insulator such as $\mathrm{Al}_{2} \mathrm{O}_{3}$ and a semi-transparent metal (about $50 \AA$ of $\mathrm{Ni}$ or $\mathrm{Au}$ ). Modulating (ac) and bias (dc) voltages are applied between the front semi-transparent metal and a contact on the back of the sample. To employ this mode the sample must be conducting.

\section{Lineshape considerations}

Differential changes in the reflectivity can be related to the perturbation of the complex dielectric function $\left[\varepsilon\left(=\varepsilon_{1}+i \varepsilon_{2}\right)\right]$ expressed as [1-3]:

$$
\Delta R / R=a\left(\varepsilon_{1}, \varepsilon_{2}\right) \Delta \varepsilon_{1}+b\left(\varepsilon_{1}, \varepsilon_{2}\right) \Delta \varepsilon_{2},
$$

where $a$ and $b$ are the Seraphin coefficients (related to the unperturbed $\varepsilon$ ) and $\Delta \varepsilon_{1} / \Delta \varepsilon_{2}$ are the changes in the complex dielectric function due to the perturbation. The functional form of $\Delta \varepsilon_{1} / \Delta \varepsilon_{2}$ can be calculated for a given perturbation provided that the dielectric function and critical point are known.

Electromodulation is the most complex form of modulation spectroscopy since it can destroy the translational symmetry of the material and hence can accelerate unbound electrons and/or holes [1-3]. Electromodulation can be classified into two categories, i.e., low- and high-field regimes. In the former $|\hbar \Theta| \leq \Gamma$, where $\Gamma$ is a broadening parameter and $\hbar \Theta$ is given by

$$
(\hbar \Theta)^{3}=e^{2} \hbar^{2} F^{2} / 2 \mu_{\|}
$$

In Eq. (2) $F$. is the field and $\mu_{\|}$is the reduced interband mass in the direction of the field. In the high-field situation $|\hbar \Theta| \geq \Gamma$.

In the low-field regime EM yields a third-derivative spectroscopy in bulk material and Eq. (1) can be written as [1-3]

$$
\Delta R / R=\operatorname{Re}\left[A \mathrm{e}^{\mathrm{i} \phi}\left(E-E_{\mathrm{g}}+\mathrm{i} \Gamma\right)^{-m}\right],
$$

where $A$ is the amplitude of the signal, $\phi$ is the phase angle which mixes together the real and imaginary parts of $E q$. (1), $E$ is the photon energy, $E_{\mathrm{g}}$ is the energy gap and $m$ is a parameter that depends on the nature of the critical point, i.e., for a three-dimensional critical point (direct band gap of GaAs) $m=2.5$. 
In the high-field regime the dielectric function of bulk material can exhibit oscillatory behavior above the band gap called Franz-Keldysh oscillations (FKOs). The position of the $n$-th extrema in the FKOs are given by [1-3]

$$
n \pi=(4 / 3)\left[\left(E_{n}-E_{\mathrm{g}}\right) / \hbar \Theta\right]^{3 / 2}+\chi,
$$

where $E_{n}$ is the photon energy of the $n$-th extrema. A plot of $(4 \pi / 3)\left(E_{n}-E_{\mathrm{g}}\right)^{3 / 2}$ vs. the index number $n$ will yield a straight line with slope $(\hbar \Theta)^{3 / 2}$. Thus, the electric field $(F)$ can be obtained directly from the period of the FKOs if $\mu_{\|}$is known.

For bound systems EM yields a first-derivative spectroscopy [1-3]

$$
\Delta \varepsilon=\left[\left(\partial \varepsilon / \partial E_{\mathrm{g}}\right)\left(\partial E_{\mathrm{g}} / \partial F_{\mathrm{ac}}\right)+(\partial \varepsilon / \partial \Gamma)\left(\partial \Gamma / \partial F_{\mathrm{ac}}\right)+\right.
$$

$$
\left.(\partial \varepsilon / \partial A)\left(\partial A / \partial F_{\mathrm{ac}}\right)\right] F_{\mathrm{ac}}
$$

where $F_{\mathrm{ac}}$ is the modulating field. For unscreened excitons (either in bulk or quantum wells) and impurities the unperturbed $\varepsilon$ of Eq. (5) will be either Lorentzian or Gaussian, depending on the broadening mechanism and/or temperature. For the Lorentzian case Eq. (5) can be conveniently expressed as Eq. (3) with $m=2$ (if the term $\partial \varepsilon / \partial A$ in Eq. (5) is neglected). For screened excitons in quantum wells the unperturbed $\varepsilon$ will be related to a broadened, step-like, two-dimensional density of states.

For thermo- and piezomodulation, since these methods do not destroy the translation symmetry, the lineshape will be the first-derivative expression of Eqs. (5) for either bound or unbound systems $[1,3]$.

\section{Applications}

\subsection{Modulation-doped pseudomorphic GaAlAs/InGaAs/GaAs single quantum wells}

Photoreflectance or CER at $300 \mathrm{~K}$ has been used to characterize $\mathrm{Ga}_{1-y} \mathrm{Al}_{y} \mathrm{As} / \mathrm{In}_{x} \mathrm{Ga}_{1-x} \mathrm{As} / \mathrm{GaAs}$ modulation-doped single quantum wells (SQWs) with a two-dimensional electron gas (2DEG) having different materials parameters $[4,5]$. Such structures form the basis for high electron mobility transistors. We shall discuss in detail one such sample with nominal $y=0.19, x=0.2$ and well width $\left(L_{\mathrm{W}}\right)=100 \AA$, which has two confined electron and two confined heavy-hole states, with the Fermi level $\left(E_{\mathrm{F}}\right)$ occurring between the first and second electron levels. Thus, four intersubband transitions, i.e., $11 \mathrm{H}, 12 \mathrm{H}, 21 \mathrm{H}$ and $22 \mathrm{H}$, are possible at room temperature. The "symmetry"-forbidden resonances $12 \mathrm{H}$ and $21 H$ are allowed because of the presence of the built-in electric field. The notation $m n H$ denotes an intersubband transition between the $m$-th conduction and $n$-th heavy-hole-like valence subbands.

Displayed by the solid line in Fig. 2 is the CER spectrum at $300 \mathrm{~K}$ originating in the InGaAs section of the sample, except for the feature denoted $E_{0}(\mathrm{GaAs})$. This resonance corresponds to the direct band gap of GaAs and originates in the GaAs buffer/substrate. The trace of Fig. 2 consists of four peaks, labelled $m n H$, riding on a background, which is due to the thermal tail of the Fermi distribution function. The dashed line in Fig. 2 is a fit to the lineshape function which is the first-derivative of a broadened, step-like, two-dimensional density of 


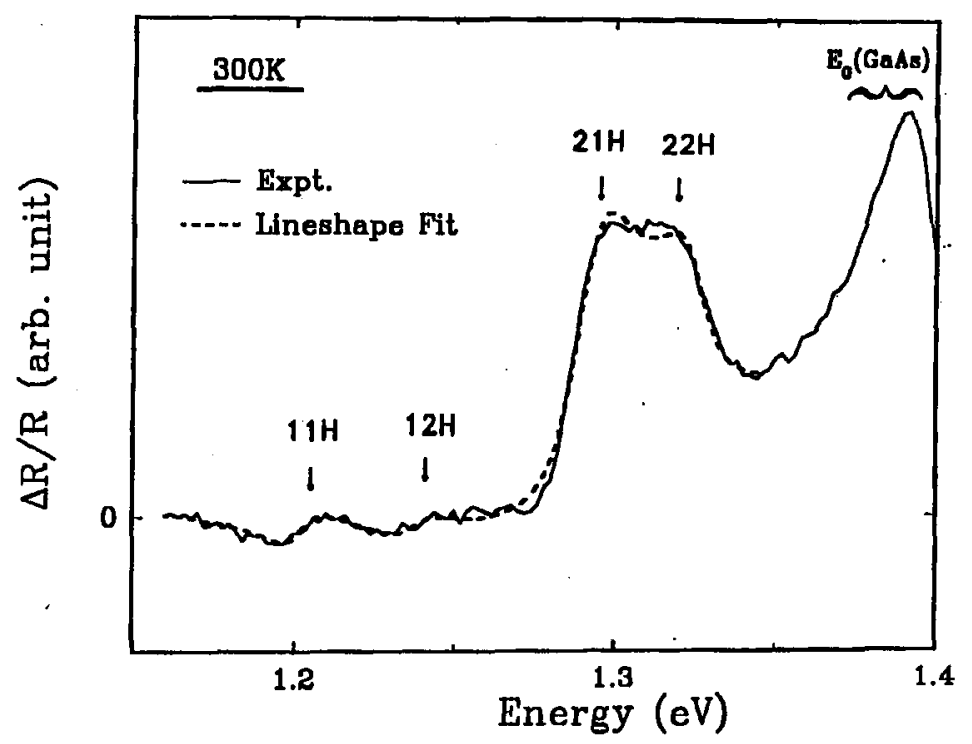

Fig. 2. The experimental room temperature CER spectrum (solid line) from the InGaAs region of a $n$-GaAlAs/InGaAs/GaAs pseudomorphic HEMT sample. The dashed line is a least squares fit to an appropriate lineshape function. The obtained values of the intersubband energies $m n H$ are designated by arrows.

states (due to the screening of the excitons by the 2DEG) and a Fermi level filling factor. The obtained energies of the $m n H$ transitions are denoted by arrows. By comparing these experimental energies with a theoretical self-consistent calculation it was possible to evaluate several important materials parameters such as $L_{\mathrm{W}}$, In composition and built-in electric field. The fit to the background yielded $E_{\mathrm{F}}$ and hence $N_{s}$.

It should be noted that at room temperature transitions to the first conduction level (i.e., $11 H$ and $12 H$ ), which lies below $E_{\mathrm{F}}$, are only seen because of the significant thermal broadening of the Fermi distribution. As the temperature is lowered these resonances are no longer observed $[4,5]$.

\subsection{Quantum well laser structures}

In spite of the proven utility of PR and CER in evaluating device structures to date little work has been performed on $\mathrm{QW}$ laser structures $[4,5]$. Low temperature $(19 \mathrm{~K})$ electroabsorption measurements have been performed on InGaAs/InGaAsP QW lasers grown lattice matched on InP substrates. A comprehensive study comparing lasing frequency with room temperature PR and PL has been performed for 24 samples of $\mathrm{Ga}_{0.2} \mathrm{Al}_{0.09} \mathrm{As}(120 \AA) / \mathrm{Ga}_{0.6} \mathrm{Al}_{0.4} \mathrm{As}(400 \AA) /$ $\mathrm{Ga}_{0.2} \mathrm{Al}_{0.8} \mathrm{As} \mathrm{QW}$ lasers. Moneger et al. (see ref. in [5]) have reported a room temperature CER study on InGaAs/GaAs/GaAlAs vertical cavity surface emitting laser (VCSEL) structures while Berger et al. (see ref. in [5]) have performed a PR study of the GaAs/GaAlAs VCSEL system. Chandler-Horowitz et al. (see ref. in [5]) 
have investigated a GaInP/AlGaInP/AlInP SQW laser structure using a double beam modulation PR technique to eliminate the PL background often encountered in PR.

Pollak et al. have recently performed a room temperature CER study of an pseudomorphic InGaAs/GaAs/InGaP $0.98 \mu \mathrm{m}$ laser structure [5]. CER was employed to eliminate PL problems and also to minimize the amount of light on the sample and hence any photovoltaic effects. Signals were detected from the InGaAs $\mathrm{SQW}, \mathrm{GaAs}$ wave guide section and $\mathrm{InGaP}$ regions of the sample. Five quantum transitions were observed from the InGaAs SQW section including $11 \mathrm{H}$, which is closely related to the lasing frequency. From a comparison of the energies of these SQW features with an envelope function calculation, including the effects of strain, the In composition and $L_{\mathrm{W}}$ of the SQW were evaluated. The derivative nature of the spectrum and the narrow linewidth made it possible to easily determine the energy position of $11 H$ to less than $1 \mathrm{~nm}$ at room temperature.

The sample used in this study was grown by OMCVD on a $n^{+}-\mathrm{GaAs}(100)$ substrate. The basic unit of the structure consisted of a pseudomorphic $\operatorname{In}_{x} \mathrm{Ga}_{1-x} \mathrm{As}$ SQW surrounded on each side by $1000 \AA$ of not-intentionally doped (NID) GaAs sandwiched between $n$-and $p$-type InGaP. From the growth conditions the intended values of the In composition and well width were $20 \%$ and $70 \AA$.

Shown by the solid line in Fig. 3 is the experimental CER data in the spectral region of the InGaAs SQW. The strong, lowest lying feature is $11 \mathrm{H}$. Above about $1.42 \mathrm{eV}$ the signal is from the GaAs. The dotted line is a least squares fit of

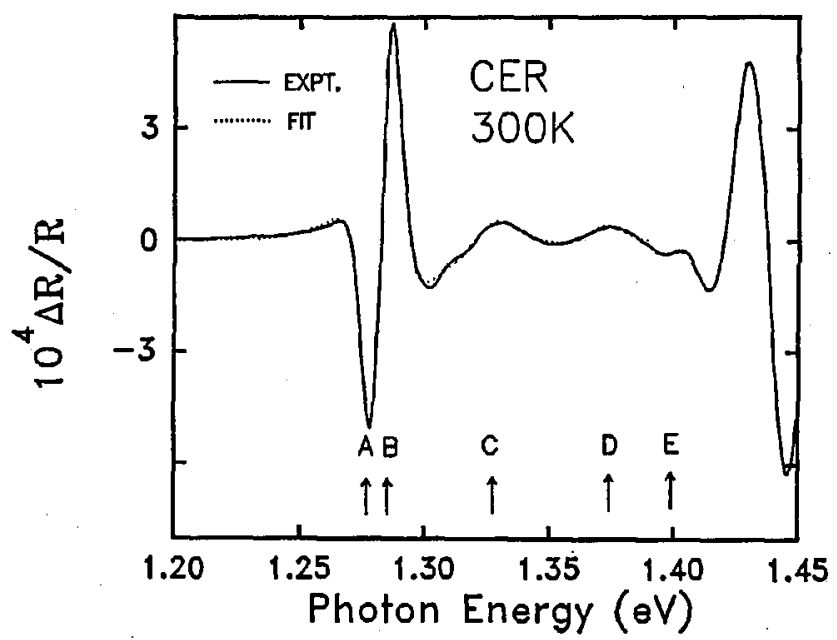

Fig. 3. The solid line is the experimental CER spectrum in the region of the InGaAs $\mathrm{SQW}$ at $300 \mathrm{~K}$. The dashed line is a least squares fit to a lineshape function, yielding values of the intersubband energies designated by arrows. 
the first-derivative of a Lorentzian profile. The obtained values of the various intersubband energies are shown at the bottom of the figure by arrows, denoted $A-D$, and also are listed in Table. There is another peak at about $1.4 \mathrm{eV}$, denoted $E$, which the authors were not able to fit because of the proximity of the GaAs signal.

\section{TABLE}

Comparison of experiment and calculation for the InGaAs $\mathrm{SQW}$ of an InGaAs/GaAs/InGaP laser structure based on an In composition of $19 \%$ and a well width of $70 \AA$.

\begin{tabular}{c|c|c|c}
\hline \hline $\begin{array}{c}\text { Spectral } \\
\text { structure }\end{array}$ & $\begin{array}{c}\text { Experiment } \\
{[\mathrm{eV}]}\end{array}$ & $\begin{array}{c}\text { Calculation }^{(a)} \\
{[\mathrm{eV}]}\end{array}$ & Transition \\
\hline$A$ & 1.279 & 1.277 & $11 H$ \\
$B$ & 1.288 & 1.310 & $12 H$ \\
$C$ & 1.325 & 1.334 & $11 L$ \\
$D$ & 1.371 & 1.359 & $21 H$ \\
$E$ & $\approx 1.4$ & 1.392 & $22 H$ \\
\hline
\end{tabular}

${ }^{(a)}$ Taking into account $7 \mathrm{meV}$ exciton binding energy.

In order to evaluate $L_{\mathrm{W}}$ and In composition of the SQW these authors compared the experimentally observed energies of the features $A-E$ with a theoretical model. They first performed an envelope function calculation, including the effects of strain to determine the band-to-band energies of the various transitions. Exciton binding energies of $7 \mathrm{meV}$ were used for the transitions. Any small shifts related to the quantum confined Stark effect, due to the built-in field, were not taken into account. The best overall agreement between the calculation and experiment was found for $L_{\mathrm{W}}=70 \AA$ and an In composition of $19 \%$, respectively, values in very good agreement with the intended growth parameters.

Displayed in Fig. 4 is the CER spectrum (solid line) in the region of the GaAs and InGaP band gaps. The GaAs signal exhibits a very large number of FKOs, accompanied by a beating pattern. From an analysis of the period of the FKOs using Eq. (4) the built-in electric field in the structure $(30 \mathrm{kV} / \mathrm{cm})$ was evaluated.

In addition, the FKOs can be used to gain information about the coherence length $(L)$ of the electron-hole pair in the NID GaAs region [6]. The length $L$ can be defined on the following basis [5]:

$$
L=\left[E_{\text {last FKo }}-E_{\mathrm{g}}(\mathrm{GaAs})\right] / q F,
$$

where $q$ is the electron charge and $F$ is the measured field $(30 \mathrm{kV} / \mathrm{cm})$. From Fig. 4 $E_{\text {last FKo }}=1.720 \mathrm{eV}$ (indicated by the arrow marked $\left.E_{\text {last FKo }}\right)$ and $E_{\mathrm{g}}(\mathrm{GaAs})=$ $1.422 \mathrm{eV}$. Therefore $L \approx 990 \AA$, i.e., about the width of the NID GaAs cladding region. This value of $L$ indicates that there is no interdiffusion of dopants into the GaAs region. 


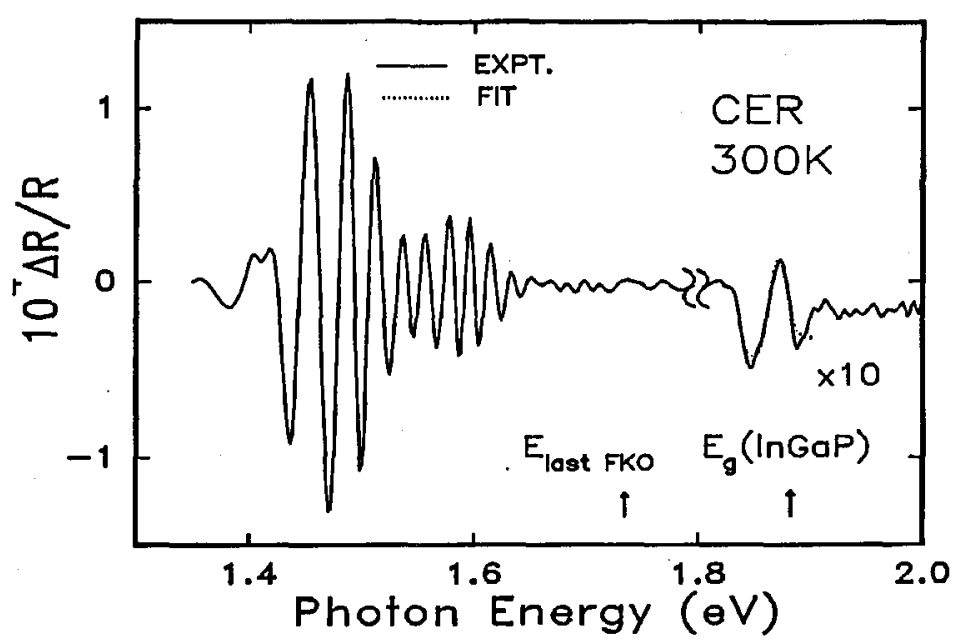

Fig. 4. The experimental CER spectrum at $300 \mathrm{~K}$ for the GaAs and InGaP regions of the sample. The arrow denotes $E_{\text {lastFko. }}$

In order to gain more information about the nature of the GaAs FKOs the authors have simulated the PR experiment performed on this device structure using a comprehensive, self-consistent model [6]. This calculation shows that the equilibrium (dark) electric field in the GaAs region is quite uniform and has a value of $65 \mathrm{kV} / \mathrm{cm}$, which is about a factor of two higher than the measured number. This difference can be accounted for on the basis of the photovoltaic (PV) effect induced by the probe beam, since there is no pump beam in CER. The magnitude of the field deduced from the calculated spectrum is $30 \mathrm{kV} / \mathrm{cm}$, about $50 \%$ smaller than the value measured from the experimental FKOs. To account for this discrepancy the authors also have included the influence of non-radiative recombination centers, which are accounted for on the basis of a Shockley-Read-Hall (SRH) term in the continuity equations for both electrons and holes. The SRH mechanism reduces the concentration of photogenerated carriers and leads to a reduction in the photovoltage. In order to estimate this effect the carrier capture times were varied over a wide range of values and spatial locations. The results of the simulation show that an enhanced SRH recombination in the vicinity of the InGaAs SQW could lead to a reduction of the observed PV effect when the carrier capture times for both the electrons $\left(\tau_{n}\right)$ and holes $\left(\tau_{p}=\tau_{n}\right)$ are about $1 \mathrm{~ns}$. One possible origin of these short recombination times are interface states at the InGaAs/GaAs interface with a density of $\approx 10^{11} \mathrm{~cm}^{-2}$. Changes in the SRH rate in the high doped regions and their interfaces $\left(0.01 \mathrm{~ns}<\tau_{\mathrm{n}}<100 \mathrm{~ns}\right)$ as well in the bulk of the NID GaAs $\left(1 \mu \mathrm{s}<\tau_{n}<1 \mathrm{~ms}\right)$ produce an insignificant increase in the calculated electric field.

The position of the observed CER peak in the region of the InGaP band gap (1.87 eV from a lineshape fit) corresponds to a lattice-matched composition ( $49 \%$ of In). 


\subsection{Quantum dot arrays}

Both undoped [6] and modulation-doped [7] quantum dot arrays have been studied using PR. We shall discuss only the former experiment. The two sets of dot array samples used in this study consisted of an $\langle 001\rangle$ semi-insulating GaAs substrate, $5000 \AA$ of a not-intentionally-doped GaAs buffer followed by either 100 periods of $\operatorname{GaAs}(80 \AA) / \mathrm{Ga}_{0.7} \mathrm{Al}_{0.3} \mathrm{As}(120 \AA)$ or 15 periods of $\mathrm{GaAs}(100 \AA) /$ $\mathrm{Ga}_{0.7} \mathrm{Al}_{0.3} \mathrm{As}(100 \AA) \mathrm{QWs}$ capped by $100 \AA$ of GaAs. The quantum dots were fabricated by reactive-ion etching. The first (second) set of samples had dot sizes of $0.5 \mu \mathrm{m}, 0.4 \mu \mathrm{m}$ and $0.23 \mu \mathrm{m}(0.5 \mu \mathrm{m}, 0.20 \mu \mathrm{m}$ and $0.10 \mu \mathrm{m})$.

From the observed behavior of the $11 H$ and $11 L$ PR features at room temperature it was possible to evaluate the process-induced strains in the quantum dots. The strain-induced shifts, $\delta E_{11 H}$ and $\delta E_{11 L}$, of the $11 \mathrm{H}$ and $11 L$ energies, respectively, can be written as (for low strains)

$$
\delta E_{11 H}=\delta E_{\mathrm{H}}+\delta E_{\mathrm{S}}, \quad \delta E_{11 L}=\delta E_{\mathrm{H}}-\delta E_{\mathrm{S}}+\ldots
$$

The hydrostatic $\left(\delta E_{\mathrm{H}}\right)$ and shear $\left(\delta E_{\mathrm{S}}\right)$ components of the strain-induced shifts in Eq. (7) are given by

$$
\delta E_{\mathrm{II}}=a\left(2 \varepsilon+\varepsilon_{z z}\right), \quad \delta E_{\mathrm{S}}=b\left(\varepsilon_{z z}-\varepsilon\right) .
$$

In Eq. (8) the parameters $a(=-8.7 \mathrm{eV})$ and $b(=-2.0 \mathrm{eV})$ are the hydrostatic and shear deformation potentials for $\mathrm{GaAs}$, respectively.

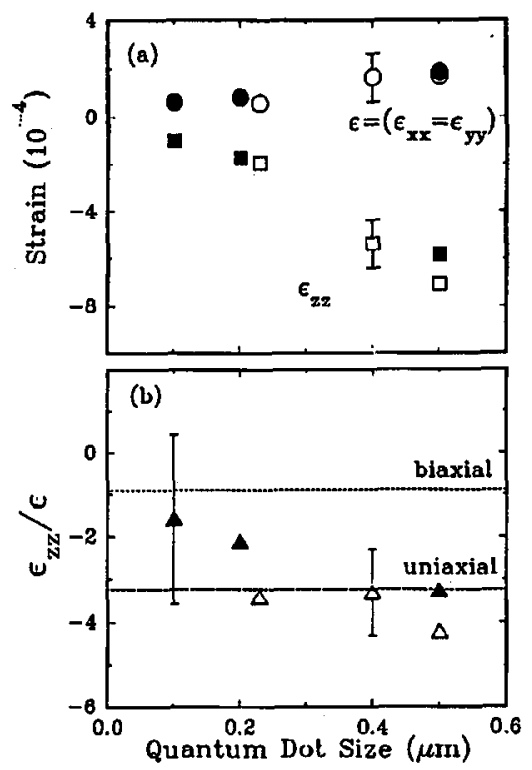

Fig. 5. (a) The process-induced strains $\varepsilon_{z z}$ and $\varepsilon=\left(\varepsilon_{x x}=\varepsilon_{y y}\right)$ and (b) the ratio $\varepsilon_{z z} / \varepsilon$ as a function of quantum dot size. Representative error bars are shown. 
From a comparison of the positions of $11 H$ and $11 L$ with Eqs. (7) and (8) it is possible to evaluate $\varepsilon\left(=\varepsilon_{x x}=\varepsilon_{y y}\right)$ and $\varepsilon_{z z}$ (along the growth direction) for the quantum dots. Plotted in Fig. 5a by the open (closed) circles and squares are the obtained values $\varepsilon$ and $\varepsilon_{z z}$, respectively, for the first (second) set of samples as a function of dot size. For the largest dot there is a compressive $\varepsilon_{z z}$ of about $-7 \times 10^{-4}$ while for the $0.10 \mu \mathrm{m}$ sample $\varepsilon_{z z}$ has been reduced to about $-1 \times 10^{4}$.

The character of the observed strain can be parameterized in terms of the ratio $\varepsilon_{z z} / \varepsilon$. For hydrostatic, biaxial and uniaxial strains this ratio can be expressed as +1 (since $\left.\varepsilon_{z z}=\varepsilon_{y y}=\varepsilon_{x x}\right),-2 C_{12} / C_{11}(=-0.9)$ and $-\left(C_{11}+C_{12}\right) / C_{12}(=-3.2)$, respectively, where the $C_{i j}$ are the elastic stiffness constants. Plotted in Fig. 5b by the open (closed) triangles is $\varepsilon_{z z} / \varepsilon$ for the first (second) set of samples. The dashed lines indicate the values of this ratio for the biaxial $(-0.9)$ and uniaxial $(-3.2)$ cases. Note that while the magnitude of the strain is decreasing with reduced dot size the nature of the strain is tending towards biaxial.

\section{Summary}

This paper has reviewed the background of modulation spectroscopy, particularly electromodulation, and has presented some recent room temperature results having both fundamental and technological significance, including two-dimensional electron gas (2DEG) effects in modulation-doped, pseudomorphic GaAlAs/ InGaAs/GaAs single quantum wells (HEMT structures), quantum well laser structures and process-induced damage in quantum dot arrays fabricated by RIE.

\section{References}

[1] F.H. Pollak, H. Shen, Mater. Sci. Eng. R 10, 275 (1993) and references therein.

[2] O.J. Glembocki, B.V. Shanabrook, Semiconductors and Semimetals, Eds. D.G. Seiler, C.L. Littler, Vol. 36, Academic Press, New York 1992, p. 222 and references therein.

[3] F.H. Pollak, in: Handbook on Semiconductors, Optical Properties of Semiconductors, Ed. M. Balkanski, Vol. 2, North-Holland, Amsterdam 1994, p. 527.

[4] F.H. Pollak, H. Qiang, D. Yan, Y. Yin, V.T. Boccio, Photonics Spectra Magazine, Vol. 27, Issue 8, August 1993, p. 78; also, J. Metals 46, 55 (1994); also, Proceedings of the ECS Symposium on Diagnostic Techniques for Semiconductor Materials and Devices, Eds. D. Schroder, J. Benton, J. Rai-Choudhury, Electrochemical Society, Pennington 1995, ECS Proc. 94-33, 228 (1994).

[5] F.H. Pollak, W. Krystek, M. Leibovitch, M.L. Gray, W.S. Hobson, to be published in IEEE Journal of Selected Topics in Quantum Electronics: Applied Optical Diagnostics of Semiconductors.

[6] H. Qiang, F.H. Pollak, Y-S: Tang, P.D. Wang, C.M. Sotomayor Torres, Appl. Phys. Lett. 64, 2830 (1994); also, Proc. SPIE 2139, 234 (1994).

[7] P.D. Wang, C.M. Sotomayor Torres, M.C. Holland, H. Qiang, F.H. Pollak, G. Gumbs, Mater. Res. Soc. Symp. Proc. 324, 187 (1994); also, G. Gumbs, D. Huang, H. Qiang, F.H. Pollak, P.D. Wang, C.M. Sotomayor Torres, M.C. Holland, Phys. Rev. B 50, 10962 (1994). 